\title{
General Discussion to papers of Dr Gschaedler et al. and Dr Kewalramani
}

DR NUSEIBEH (G.B.). In I 975 we surveyed one hundred consecutive patients who were admitted to the Spinal Unit here and we found that 7 per cent of them did have gastro-intestinal bleeding. Now we found that five out of the seven had associated head injury which makes one wonder that it has got some element in it. The second thing which I would like to elaborate on is the question of drugs. They were all having anticoagulant therapy, so is the anticoagulant therapy an ulcerogenic drug or not. This is something to be looked at. A very important point also, in the first few days the patient complains of back ache or shoulder ache or what have you and the nurse or the junior doctor give him aspirin or some similar drug, and these are well known ulcerogenic drugs. These are precipitating factors which we found in our series.

DR HACHEN (Chairman). Thank you. Before giving the microphone to Dr Kewalramani I would like to say that with regard to anticoagulation, in Geneva we have all our patients on two daily doses of 10000 units of heparin beginning usually about 12 hours after injury, with the exception of comatose patients. In none of those cases have we had any ulcerations, the patients may have had erosions but we have never had any clinical signs of ulceration during the last 5 years. Since we are using systematic anticoagulation we have never had any bleeding ulcer. So that I think that this anticoagulation creates no major hazard to these patients. What is most important is accurate initial nursing and sedation and I think that many patients who pass through intensivecare stations where they are exposed to all the machinery and equipment of re-animation may have increased stress, and I think also that one should not inform them about the definite state of their spinal cord injury before at least 2 to 3 weeks after the injury. Dr Kewalramani, would you like to give your comment.

Dr Kewalramani. I definitely agree with Dr Silver that the incidence is definitely higher than what is often reported. I think most of us probably in the past have accepted as a natural course of events that gastro-intestinal bleeding is fairly frequent and I think we need to take a second look at that in an attempt to come up with somewhat more definite numbers and somewhat more definite figures. Now any ulcerogenic drugs I tried to look at in these 20 patients, a number of patients were on Davon or Tywen in the beginning as pain relievers. Now, as far as I know, these two drugs I am unaware of being implicated as ulcerogenic drugs, so at least I cannot draw any conclusions from these 20 patients.

Dr SIIVER (G.B.). Dr Dollfus obviously saw his acute traumatic cases in an acute hospital though he was unlucky that he was really in the firing line. Fortunately, we were not in the firing line, but we've had three patients who have survived traumatic ruptures of the aorta and come to us as paraplegics, but we obviously were seeing them when they'd been through a thoracic unit. One of them was a fascinating case, a traumatic rupture. Another one was a boy who had a ruptured aorta and the rupture was repaired and when the rupture was repaired the boy became paraplegic, so they are not quite the same as your group. With regard to the second paper about the gastrointestinal bleeding; of the IOO cases that Mr Nuseibeh referred to, it is interesting that several of them, after they bled, were questioned more closely, and they told us that they had in fact had pain in their abdomen prior to injury, they probably had a previous history of a duodenal ulcer, and when you bear in mind particularly the motor cyclist and the young people and the irregular lives that they lead with their heavy drinking, smoking and absence of meals, I think this is an important factor to be considered in the aetiology of these ulcerations. They have a pre-existing ulcer which becomes exacerbated by, the first 48 hours they don't take very much by mouth and they are distended, and I think this can flare up an ulcer and cause bleeding.

DR Dollfus (France). Recently in Paris we've had a very good meeting on paraplegia and Guido Zäch, Chief of the Swiss Paraplegic Centre of Basle, has made a very important study using systematic gastroscopic examination in tetraplegics and paraplegics 
and has found that there is a very high incidence of ulcers, bleeding or not, in the complete cervical group compared with the incomplete cervical group and as far as you go down in the level of the lesion the less you see these ulcers. Most of these patients develop these gastro-duodenal ulcers about Io to 12 days after injury.

DR HAChEN. I see. Otherwise as we go on to this paper or to these remarks, I wonder to what point repeated gastroscopy in such patients may not be in itself an ulcer-inducing factor. This might considerably modify the results.

DR Dollfus. May I add something else on a personal basis and that is I join you completely as we are using anticoagulant therapy from the start and we have a very low incidence of gastro-intestinal bleeding. 